\title{
Aufklärung und Einwilligung des Patienten in die ärztliche Behandlung - eine Übersicht für Urologen unter Berücksichtigung des neuen Betreuungsrechtes
}

\author{
K. Wasserburg, Rechtsanwalt \\ Mainz
}

\section{Einführung}

Am 1.1.1992 trat das Gesetz zur Reform des Rechts der Vormundschaft für Volljährige in Kraft ${ }^{1}$. Nach diesem Gesetz sollen Kranke und gebrechliche Personen nicht mehr länger ausgegrenzt und entrechtet sein, wie es nach den bisherigen über neunzig Jahre alten Regelungen im BGB der Fall war. Ziel des Gesetzes ist daher, diesen Menschen eine durch Hilfe unterstützte Einbeziehung in das Rechtsleben zu gewährleisten. Durch das Gesetz wurden zahlreiche Vorschriften des BGB geändert oder neu eingeführt, die auch für den ärztlichen Tätigkeitsbereich - vor allem bei der Behandlung von psychisch Kranken und alten Menschen - von Bedeutung sind. Wichtigstes Kennzeichen des Gesetzes ist die Abschaffung der bisherigen Institute der Entmündigung, Vormundschaft und Pflegschaft. An ihre Stelle tritt ein sog. Betreuer, der bestellt wird, wenn „ein Volljähriger aufgrund einer psychischen Krankheit oder einer körperlichen, geistigen oder seelischen Behinderung seine Angelegenheiten ganz oder teilweise nicht besorgen" kann².

\section{Untersuchung des Gesundheits- zustandes, Heilbehandlung und ärztlicher Eingriff}

Die Rechtsstellung des Betreuers bringt Neuerungen bei der Aufklärung und der Einwilligung im Rahmen der allgemeinen ärztlichen Behandlung; darüber hinaus sind aber auch besondere Vorschriften, z. B. bei der Sterilisation, zu beachten (dazu i.E. unten III).

\section{Der einwilligungsfähige Patient}

Die gesetzliche Neuregelung knüpft an den bisher schon in Rechtsprechung und Literatur entwickelten Begriff der "Einwilligungsfähigkeit" an und übernimmt ihn (\$ 1905 BGB) in das Gesetz ${ }^{3}$. Einwilligungsfähig ist ein Patient, wenn er die natürliche Einsichts- und Steuerungsfähigkeit besitzt, um Bedeutung und Tragweite eines ärztlichen Eingriffes - nach entsprechender Aufklärung - zu erkennen und gemä $\beta$ dieser Erkenntnis zu urteilen ${ }^{4}$.

Diese Fähigkeit hat der behandelnde Arzt sorgfältig zu prüfen; gelangt er zu der Überzeugung, da $\beta$

Akt. Urol. 23 (1992) 325-327

(c) Georg Thieme Verlag Stuttgart · New York die natürliche Einsichtsfähigkeit des Patienten im Hinblick auf die konkrete Krankheit und die ins Auge gefaßte Maßnahme vorliegt, so kann auch ein psychisch kranker Patient wirksam in die Behandlung einwilligen, selbst wenn ihm ein Betreuer bestellt ist. Darum ist auch nur er aufzuklären. Diese bisher bestehende Rechtslage wird auch durch das Betreuungsgesetz nicht verändert, sondern übernommen; in bestimmter Hinsicht wird aber die Prüfungspflicht des behandelnden Arztes verschärft: dadurch, daß das Institut der Entmündigung abgeschafft wurde, erhöht sich für ihn der Zwang zu individueller Prüfung. Während er bislang annehmen konnte, daß einem Entmündigten in aller Regel auch die Einwilligungsfähigkeit fehlt, hat er nun keinen Anhaltspunkt durch eine bestimmte gerichtliche Entscheidung mehr. Selbst wenn er weiß, daß dem Patienten ein Betreuer bestellt wurde, hilft ihm das nicht weiter, weil sich dessen Aufgabenkreis nach dem neuen Recht auf die Angelegenheiten beschränkt, in denen eine Betreuung erforderlich ist (§ 1896 II BGB).

Wie bisher ist von der Einwilligungsfähigkeit scharf die Geschäftsfähigkeit zu unterscheiden: Letztere betrifft nur die Wirksamkeit des zivilrechtlichen Arztvertrages. Einwilligungsfähigkeit und Geschäftsfähigkeit müssen sich nicht decken. Auch ein geschäftsunfähiger Erwachsener kann unter Umständen einen einfachen ärztlichen Eingriff, wie z. B. das Ziehen eines Zahnes, verstehen und darin einwilligen. Die Abschaffung der Entmündigung hat aber für den Abschluß des Arztvertrages eine Veränderung mit sich gebracht: Ein unter Betreuung stehender kranker Mensch bleibt grundsätzlich weiterhin geschäftsfähig, es sei denn, er befindet sich in einem die freie Willensbestimmung ausschließenden Zustand krankhafter Störung der Geistestätigkeit ( $\$ 104$ Nr. 2 BGB). Ferner kann nunmehr ein Vertragsschluß durch ihn von der Einwilligung des Betreuers abhängen, wenn das Vormundschaftsgericht das besonders angeordnet hat $(\$$ 1903 BGB, ähnlich der beschränkten Geschäftsfähigkeit bei Kindern). Beides ist nicht leicht für den aufnehmenden Arzt feststellbar.

\footnotetext{
1 Betreuungsgesetz - BGBl. I, S. 2002.

2 Eine besonders für Ärzte gute Darstellung des Betreuungsrechts bei: Schmidt, G./Böcker, F.: Betreuungsrecht. Eine systematische Einführung aus juristischer und psychiatrischer Sicht, 1. Aufl., München 1991.

3 BT Drucks 11/4528. Begr. S. 71, 140.

4 vgl. zur Entwicklung des Begriffes etwa: Kern/Laufs, Die ärztliche Aufklärungspflicht 1983, S. 24 ff; Gießen, Arzthaftungsrecht 1990, S. 128 m.w. N.
} 


\section{Der nicht einwilligungsfähige Patient}

Gelangt der Arzt zu der Überzeugung, der Patient sei in dem dargestellten Sinne nicht einwilligungsfähig, so ist bei der Frage, wie er eine wirksame Ersatzeinwilligung für den Patienten erreichen kann, danach zu unterscheiden, ob dem Patienten bereits ein Betreuer bestellt ist oder nicht.

a) Ist dem Patienten bereits ein Betreuer bestellt worden, so kann dieser nach $\$ 1902$ BGB als gesetzlicher Vertreter grundsätzlich für den Betroffenen wirksam in die Heilbehandlung einwilligen. Insoweit ähnelt seine Stellung der des bisherigen Vormundes oder des Pflegers. Drei wichtige Neuerungen und Einschränkungen dieses Grundsatzes sind aber zu beachten:

aa) Das neue Recht sieht (\$ 1901 I 2, II BGB) vor, da der Betreuer bei seinen Entscheidungen so weit als möglich die Wünsche des Betreuten (des Patienten) zu berücksichtigen hat oder wie das Gesetz treffend formuliert: „Zum Wohl des Betreuten gehört auch die Möglichkeit, im Rahmen seiner Fähigkeiten sein Leben nach seinen eigenen Wünschen und Vorstellungen zu gestalten“. Der verantwortliche Betreuer muß darum alle wichtigen Entscheidungen mit dem Betreuten besprechen. Das setzt aber voraus, daß beide, wenn es um eine Heilbehandlung geht, Grund und Tragweite des ärztlichen Tuns kennen müssen. Das hat aber für den Arzt zur Folge, daß er auch bei der Behandlung eines nicht einwilligungsfähigen Erwachsenen auf jeden Fall beide - den Patienten und seinen Betreuer - aufzuklären hat.

bb) Da ein Betreuer immer nur für einen bestimmten Aufgabenkreis bestellt wird ( $\$ 1896$ II 1 BGB), kann es sein, daß einem nicht einwilligungsfähigen $\mathrm{Pa}-$ tienten zwar ein Betreuer bestellt wird, dieser aber nicht für die Heilbehandlung zuständig ist, er also nicht wirksam einwilligen kann. Der Arzt oder der Betreuer müssen in diesem Fall beim Vormundschaftsgericht die Erweiterung des Aufgabenkreises des Betreuers (nach \$ 1908 d III BGB) beantragen. Dieser Umstand zwingt den Arzt nicht einfach auf eine Einwilligung des Betreuers zu vertrauen, sondern auch dazu immer nachzufragen, ob ärztliche Behandlungen von dessen Aufgabenkreis überhaupt umfaßt sind.

cc) Eine besonders wichtige Neuerung des Betreuungsgesetzes besteht darin, daß für bestimmte, besonders gefährliche Maßnahmen des Arztes die Einwilligung des Betreuers nicht ausreicht. § 1904 BGB schreibt vor, daß für eine Untersuchung des Gesundheitszustandes, eine Heilbehandlung oder einen ärztlichen Eingriff der Betreuer der Zustimmung des Vormundschaftsgerichtes bedarf, wenn die begründete Gefahr besteht, daß der Patient aufgrund der Maßnahme stirbt oder einen schweren und längerdauernden gesundheitlichen Schaden erleiden kann. Gedacht ist dabei etwa an eine Risikooperation an herzkranken Patienten ${ }^{5}$ oder an einen Eingriff im Genitalbereich, der die Gefahr des Verlustes der Gebär- oder Zeugungsfähigkeit mit sich bringt. Die Genehmigung ist nur dann nicht erforderlich, wenn mit dem Aufschub der Maßnahme Gefahr verbunden ist ( $\$ 1904$ S. 2 BGB).
Das Verfahren zur Erlangung dieser vormundschaftlichen Genehmigung bei schweren und gefährlichen Eingriffen ist überaus kompliziert: Der betroffene Patient ist persönlich anzuhören und das Gericht $m u ß$ ein Sachverständigengutachten über die Notwendigkeit des Eingriffs, mögliche Alternativen und den Grad der Gefährlichkeit einholen. Dabei muß der Sachverständige vom behandelnden Arzt verschieden sein ( $\$ 69$ d II FGG). Bedenklich ist, daß das Gericht ohne weiteres nähere Verwandte des Patienten (Eltern, Pflegeeltern, Kinder, Ehegatte) zu der Entscheidung hören soll ( $\$ 69$ d II 2, 68 a FGG). Dadurch besteht nämlich die Möglichkeit, daß diese Kenntnis von den Leiden des Patienten erhalten, ohne daß er das will. Sein Selbstbestimmungsrecht wird an versteckter Stelle eingeschränkt. Sachgerechter hätte man das so regeln sollen wie bei den anderen Vertrauenspersonen: Sie werden auf Verlangen des Patienten gehört.

Ein Arzt, der an einem nicht einwilligungsfähigen Patienten einen besonders risikoreichen Eingriff vornehmen will, wird darum künftig immer dessen Betreuer auf die Notwendigkeit der vormundschaftsgerichtlichen Genehmigung hinweisen und deren Erteilung - außer bei Gefahr im Verzug abwarten müssen ${ }^{6}$.

b) Ist dem einwilligungsunfähigen Patienten noch kein Betreuer bestellt worden, so muß der Arzt, um eine wirksame Einwilligung zu erreichen, beim Vormundschaftsgericht die Bestellung eines Betreuers anregen. Das Gericht wird dann von Amts wegen ein Verfahren einleiten, in welchem es die Notwendigkeit der Bestellung des Betreuers prüfen und entsprechend entscheiden wird. Dabei wird unter anderem der betroffene Patient gehört und ein Sachverständigengutachten eingeholt ${ }^{7}$. Ist der Betreuer bestellt worden, so gelten die oben unter 2 a dargestellten Grundsätze.

Ist die nach der Überzeugung des Arztes zu treffende Maßnahme so dringend, daß der Abschluß dieses Verfahrens nicht abgewartet werden kann, so gelten (wie nach bisherigem Recht) die Grundsätze des rechtfertigenden Notstandes: Ein ohne wirksame Einwilligung beim einwilligungsunfähigen Patienten durchgeführter Eingriff ist rechtmäßig, wenn er erforderlich und eine nicht anders abwendbare Gefahr für dessen Leib und Leben $\mathrm{zu}$ verhindern war $^{8}$.

\section{Zweifel an der Einwilligungsfähigkeit des Patienten}

Häufig werden auch nach einem gründlichen Gespräch mit dem Patienten beim Arzt Zweifel darüber bleiben, ob er einwilligungsfähig ist oder nicht. Für diese Situation gibt das Betreuungsrecht dem Arzt leider keine Hilfe. Ist dem Patienten bereits ein Betreuer bestellt, so ist ihm zu raten, „sicherheitshalber“ auch diesen auf-

\footnotetext{
5 Zimmermann/Damrau NJW 1991/538, 541.

6 Näheres zur Frage, wann ein Eilfall vorliegt mit Beispielen bei: Schmidt/Böcker, aaO, S. $180 \mathrm{f}$.

7 i. E. zu diesem Verfahren Zimmermann/Damrau NJW 1991, $538,542 \mathrm{ff}$.

8 Lenckner, in: Schönke/Schröder, StGB, § 34 Anm. 8.
} 
zuklären und seine Einwilligung einzuholen ${ }^{9}$. Das führt aber dann zu Schwierigkeiten, wenn Patient und Betreuer verschiedene Entscheidungen treffen - der eine willigt ein, der andere nicht. Dann bleibt wieder beim Arzt die Entscheidung, ob er nicht doch den Patienten für so verständig hält, daß er seiner Entscheidung folgen soll. Ist dem Patienten im Falle von Zweifeln über die Einwilligungsfähigkeit noch kein Betreuer bestellt, dann sollte er auf jeden Fall das unter $2 \mathrm{~b}$ beschriebene Verfahren beim Vormundschaftsgericht einleiten, weil das Gericht auch prüfen wird, ob der Betroffene für die fragliche ärztliche Maßnahme einwilligungsfähig ist.

\section{Einwilligung in eine Sterilisation}

Für den Bereich der Urologie von besonderem Interesse ist, daß für die Sterilisation eine eigene Regelung getroffen wurde.

\section{Die Sterilisation Minderjähriger}

Der neu eingeführte $\$ 1631$ c BGB trifft zur Sterilisation eine unmißverständliche Regelung: Die Sterilisation Minderjähriger ist grundsätzlich unzulässig. Weder die Einwilligung der Eltern, noch die eines Pflegers können einen solchen Eingriff rechtfertigen. Unter Juristen ist allerdings fraglich, ob diese Regelung mit dem Grundgesetz vereinbar ist, weil sie das elterliche Sorgeund Erziehungsrecht so stark einschränkt, daß selbst ein geistig schwer behindertes Kind, für das eine Schwangerschaft eine Verschlimmerung des Leidens bedeuten würde, nicht sterilisiert werden $\operatorname{darf}^{10}$.

\section{Die Sterilisation einwilligungsfähiger Erwachsener}

Hier gilt weiterhin, daß ein einwilligungsfähiger Erwachsener wirksam in eine Sterilisation einwilligen kann, auch wenn ein eventuell bestellter Betreuer dem widerspricht ${ }^{11}$.

\section{Die Sterilisation nicht einwilligungs- fähiger Erwachsener}

Hier macht der neu geschaffene $§ 1905$ BGB die Wirksamkeit einer Einwilligung des Betreuers von vielen Voraussetzungen und zusammen mit dem Gesetz über die freiwillige Gerichtsbarkeit (FGG) einem langen Verfahren abhängig.

Zunächst muß, wenn eine Sterilisation vorgenommen werden soll, ein besonderer Betreuer nur für diesen Fall bestellt werden (Sterilisationsbetreuer, $\$ 1899$ II BGB).

Dieser darf nur dann wirksam in die Sterilisation einwilligen, wenn der Betreute auf Dauer einwilligungsunfähig bleiben wird und die Sterilisation seinem (natürlichen) Willen nicht widerspricht. Ferner muß eine Schwangerschaft die Gefahr einer schwerwiegenden körperlichen oder seelischen Beeinträchtigung der Schwangeren in sich bergen und eine Schwangerschaft darf nicht durch andere zumutbare Mittel verhindert werden können (vgl. § 1905 I BGB).

Gibt der Sterilisationsbetreuer aufgrund dieser Kriterien seine Zustimmung, so ist deren Wirksamkeit weiterhin von der Genehmigung des Vormundschaftsgerichtes abhängig ( $\$ 1905$ II 1 BGB). Das Gericht muß mindestens zwei Sachverständigengutachten über insgesamt fünf Gebiete (medizinische, psychologische, soziale, sonderpädagogische und sexualpädagogische Gesichtspunkte) einholen (\$ 69 d III 3 FGG). Die Sachverständigen dürfen wiederum mit den behandelnden Ärzten nicht identisch sein. Ferner soll der Richter nahen Angehörigen und auf Verlangen anderen Vertrauenspersonen Gelegenheit zur Äußerung geben und er soll mit dem Betroffenen ein Schlußgespräch führen. Im Falle einer positiven Entscheidung darf die Sterilisation erst zwei Wochen nach deren Zugang beim Sterilisationspfleger und beim Bevollmächtigten des Betroffenen durchgeführt werden.

Es bleibt zu hoffen, daß sich der Wille des Gesetzgebers, nicht einwilligungsfähige Menschen bei einer Sterilisation besonders zu schützen, trotz dieser komplizierten Regelung in der Praxis verwirklichen läßt.

Rechtsanwalt Dr. Klaus Wasserburg

Holzstraße 42

$6500 \mathrm{Mainz}$

\footnotetext{
9 so Zimmermann/Damrau NJW 1991, 538, 540.

10 vgl. etwa Soergel-Damrau \$ 1631 c Anm. 4.

11 vgl. Zimmermann/Damrau NJW 1991, 541.
} 